TITLE:

\title{
Kinetic model of thermophilic L- lactate fermentation by Bacillus coagulans combined with real-time PCR quantification.
}

\section{$\operatorname{AUTHOR}(\mathrm{S})$ :}

Hidaka, T; Horie, T; Akao, S; Tsuno, H

\section{CITATION:}

Hidaka, T ... [et al]. Kinetic model of thermophilic L-lactate fermentation by Bacillus

coagulans combined with real-time PCR quantification.. Water research 2010, 44(8): 25542562

ISSUE DATE:

2010-04

URL:

http://hdl.handle.net/2433/120954

\section{RIGHT:}

(c) 2010 Elsevier B.V.; This is not the published version. Please cite only the published version.; この論文は出版社版でありません。引用の際に は出版社版をご確認ご利用ください。 
Table 1 Reaction rate and mass balance for model development

(Reaction rate)

Degradation of particulate carbohydrate (gCOD/(L·hr)):

$$
R_{1}=k_{1} \cdot \frac{C_{C} / X}{K_{C_{c}}+C_{C} / X} \cdot X
$$

Degradation of soluble carbohydrate (gCOD/(L·hr)): $R_{2}=k_{2} \cdot \frac{S_{C}}{K_{S_{C}}+S_{C}} \cdot X$

Lactate fermentation (gCOD/(L·hr)):

$$
R_{3}=k_{3} \cdot \frac{S_{G}}{K_{S_{G}}+S_{G} \cdot\left(1+\frac{S_{G}}{K_{E S}}\right)} \cdot \frac{K_{E I}}{K_{E I}+S_{L}} \cdot \frac{K_{E I N}}{K_{E I N}+S_{N}} \cdot\left(1-\frac{\max \left(0, S_{L}-S_{L c t}\right)}{S_{L \max }-S_{L c t}}\right) \cdot X
$$

Self-degradation (gCOD/(L·hr)): $R_{4}=k_{4} \cdot X$

(Mass balance)

$$
\begin{aligned}
& \frac{d C_{C}}{d t}=-R_{1} \\
& \frac{d S_{C}}{d t}=R_{1}-R_{2} \\
& \frac{d S_{G}}{d t}=R_{2}-R_{3} \\
& \frac{d S_{L}}{d t}=(1-Y) \cdot R_{3} \\
& \frac{d X}{d t}=Y \cdot R_{3}-R_{4}
\end{aligned}
$$

$k_{1}$, Rate constant for $\mathrm{R}_{1}(\mathrm{gCOD} /(\mathrm{gCOD} \cdot \mathrm{hr})) ; \quad k_{2}$, Rate constant for $\mathrm{R}_{2}(\mathrm{gCOD} /(\mathrm{gCOD} \cdot \mathrm{hr})) ; k_{3}$, Rate constant for $\mathrm{R}_{3}$ (gCOD/(gCOD·hr)); $k_{4}$, Rate constant for $\mathrm{R}_{4}(1 / \mathrm{hr}) ; K_{C_{c}}$, Half saturation constant for $\mathrm{R}_{1}(\mathrm{gCOD} / \mathrm{L}) ; K_{S_{C}}$, Half saturation constant for $\mathrm{R}_{2}(\mathrm{gCOD} / \mathrm{L}) ; K_{S_{G}}$, Half saturation constant for $\mathrm{R}_{3}$ (gCOD/L); 
$K_{E S}$, Inhibition parameter of glucose for $\mathrm{R}_{3}(\mathrm{gCOD} / \mathrm{L}) ; K_{E I}$, Inhibition parameter of lactate for $\mathrm{R}_{3}(\mathrm{gCOD} / \mathrm{L}) ; K_{E I N}$, Inhibition parameter of $\mathrm{NaCl}$ for $\mathrm{R}_{3}(\mathrm{gNaCl} / \mathrm{L}) ; S_{L c t}$, Lactate concentration when inhibition of lactate starts (gCOD/L); $S_{L \max }$, Maximum lactate concentration (gCOD/L); Y, Yield constant for $\mathrm{R}_{3}$ (gCOD/gCOD); 
Table 2 Experimental condition

\begin{tabular}{lcccccccccc}
\hline Run & & $1 \mathrm{a}$ & $1 \mathrm{~b}$ & 2 & 3 & 4 & 5 & 6 & 7 & 8 \\
\hline Glucose & $(\mathrm{g} / \mathrm{L})$ & 10 & 10 & 10 & 10 & 50 & 50 & 100 & 0 & 0 \\
\hline Lactate & $(\mathrm{g} / \mathrm{L})$ & - & - & 20 & - & - & 6 & - & - & - \\
\hline NaCl & $(\mathrm{g} / \mathrm{L})$ & - & - & - & 20 & - & - & - & - & - \\
\hline Kitchen garbage & $(\mathrm{L} / \mathrm{L})$ & - & - & - & - & - & - & - & 0.5 & 0.25 \\
\hline Banana peel & $(\mathrm{L} / \mathrm{L})$ & - & - & - & - & - & - & - & - & 0.17 \\
\hline
\end{tabular}


Table 3 Characteristics of kitchen garbage

\begin{tabular}{lcccc}
\hline & \multicolumn{2}{c}{ Kitchen garbage } & \multicolumn{2}{c}{ Banana peels } \\
\cline { 2 - 5 } & Total & Soluble & Total & Soluble \\
\hline TS (g/L) & 190 & - & 121 & - \\
\hline SS (g/L) & 86 & - & 65 & - \\
\hline VTS (\%) & 96 & - & 88 & - \\
\hline COD (g/L) & 201 & 114 & 79 & 42 \\
\hline Carbohydrate (g/L as glucose) & 115 & 96 & 44 & 33 \\
\hline Protein (g/L as albumin) & 20.3 & 3.7 & 4.5 & 1.6 \\
\hline T-N (mgN/L) & 3.4 & 1.2 & 1.4 & 1.0 \\
\hline pH (-) & 5.1 & - & 5.5 & - \\
\hline
\end{tabular}


Table 4 Summary of each batch experiment

\begin{tabular}{lcccccccccc}
\hline Run & & $1 \mathrm{a}$ & $1 \mathrm{~b}$ & 2 & 3 & 4 & 5 & 6 & 7 & 8 \\
\hline Time when fermentation started & $(\mathrm{h})$ & 6 & 6 & 20 & 11 & 6 & 7 & 9 & 12 & - \\
\hline Lactate concentration at the end & $(\mathrm{g} / \mathrm{L})$ & 8.6 & 9.3 & 29 & 9.3 & 45 & 48 & 52 & 35 & $21.5 \pm 0.4$ \\
\hline $\begin{array}{l}\text { Lactate production ratio } \\
\text { from carbohydrate }\end{array}$ & $(-)$ & 0.86 & 0.93 & 0.74 & 0.89 & 0.90 & 0.84 & 0.52 & 0.54 & 0.59 \\
\hline OP & $(\%)$ & 100 & 100 & 100 & 100 & 100 & 100 & 98.3 & 98.5 & 99.3 \\
\hline
\end{tabular}


Table 5 Model parameters

\begin{tabular}{cc}
\hline parameter & value \\
\hline$k_{1}$ & $0.1(\mathrm{gCOD} /(\mathrm{gCOD} \cdot \mathrm{hr}))$ \\
\hline$k_{2}$ & $80(\mathrm{gCOD} /(\mathrm{gCOD} \cdot \mathrm{hr}))$ \\
\hline$k_{3}$ & $12.8(\mathrm{gCOD} /(\mathrm{gCOD} \cdot \mathrm{hr}))$ \\
\hline$k_{4}$ & $1.0(\mathrm{gCOD} / \mathrm{L})$ \\
\hline$K_{C_{c}}$ & $1.0(\mathrm{gCOD} / \mathrm{L})$ \\
\hline$K_{S_{C}}$ & $3.1(\mathrm{gCOD} / \mathrm{L})$ \\
\hline$K_{S_{G}}$ & $180(\mathrm{gCOD} / \mathrm{L})$ \\
\hline$K_{E S}$ & $5.11(\mathrm{gCOD} / \mathrm{L})$ \\
\hline$K_{E I}$ & $30(\mathrm{gNaCl} / \mathrm{L})$ \\
\hline$K_{E I N}$ & $40(\mathrm{gCOD} / \mathrm{L})$ \\
\hline$S_{L c t}$ & $56(\mathrm{gCOD} / \mathrm{L})$ \\
\hline$S_{L \max }$ & $0.12(\mathrm{gCOD} / \mathrm{gCOD})$ \\
\hline$Y$ & \\
\hline$Y$ & \\
\hline
\end{tabular}




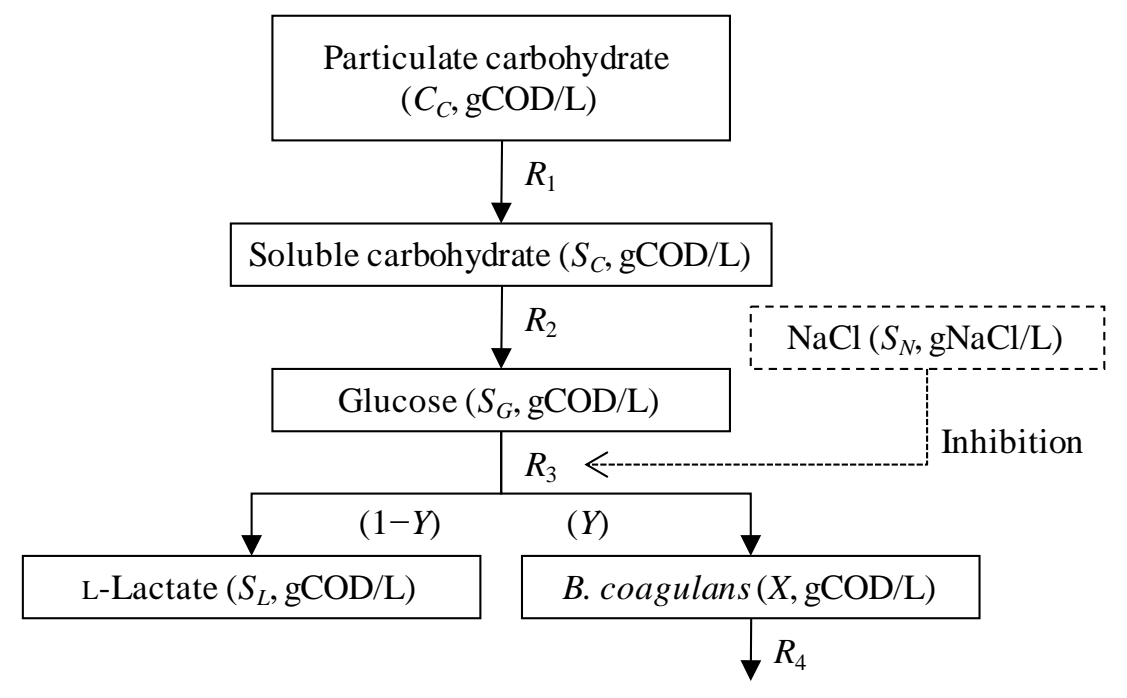

Figure 1 State variables and transformation paths 

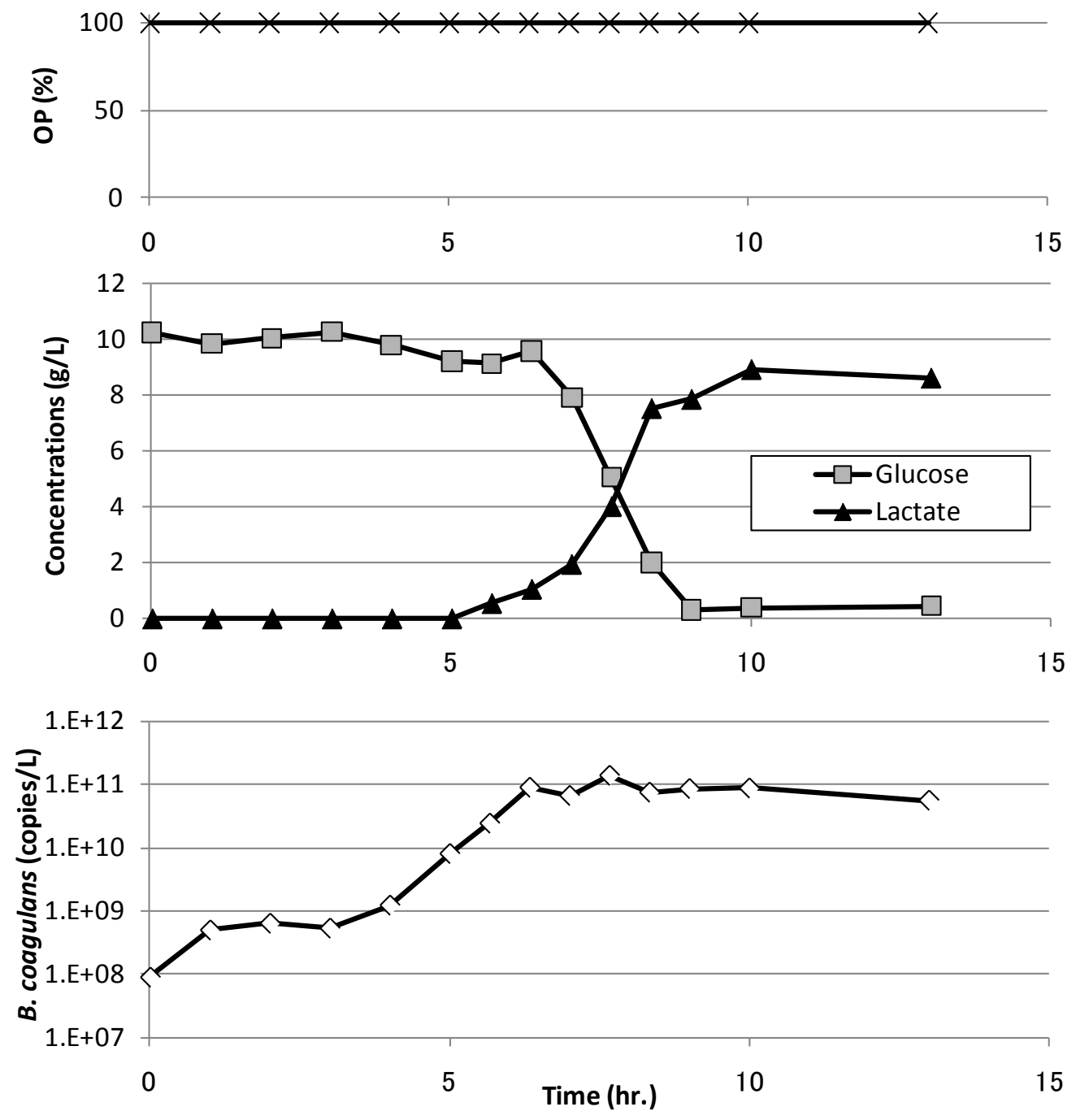

Figure 2 Results of Run 1a 


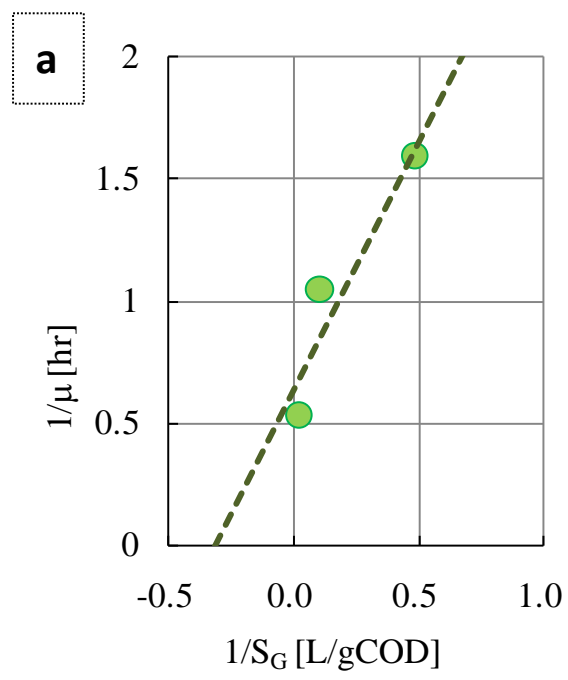

$$
\begin{array}{ll}
\Delta & \mathrm{S}_{\mathrm{L}} \geq 50 \\
\times & \mathrm{S}_{\mathrm{L}}=41 \sim 48 \\
\square & \mathrm{S}_{\mathrm{L}}=28 \sim 32 \\
\Delta & \mathrm{S}_{\mathrm{L}}=14 \sim 24 \\
\bigcirc & \mathrm{S}_{\mathrm{L}}=0 \sim 10
\end{array}
$$
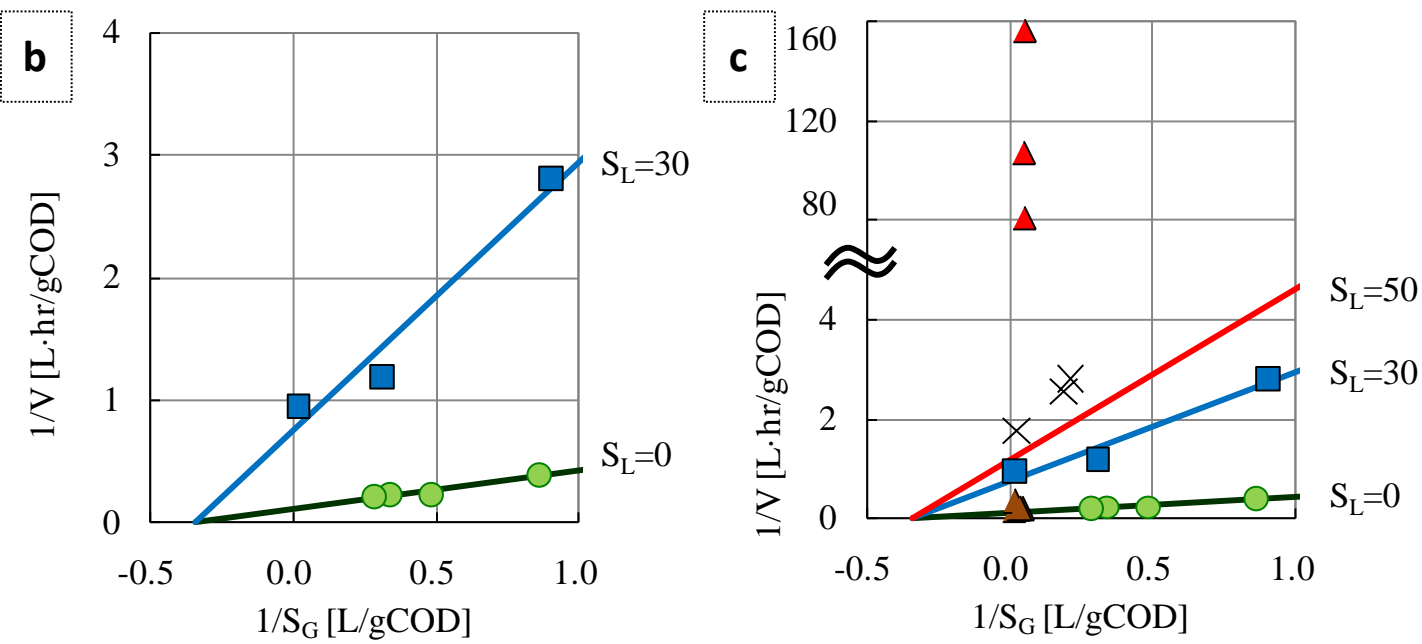

Figure 3 Lineweaver-Burk plot for accumulated lactate inhibition depending on glucose concentrations. $\mu$ is specific growth rate $(1 / \mathrm{hr}), \mathrm{V}$ is measured glucose consumption rate (gCOD/(L·hr)), $\mathrm{S}_{\mathrm{G}}$ and $\mathrm{S}_{\mathrm{L}}$ are concentrations of glucose and lactate, respectively. Solid lines are calculated by the non-competitive inhibition model equation when $\mathrm{S}_{\mathrm{G}}$ is 0,30 , or 50 (gCOD/L). 


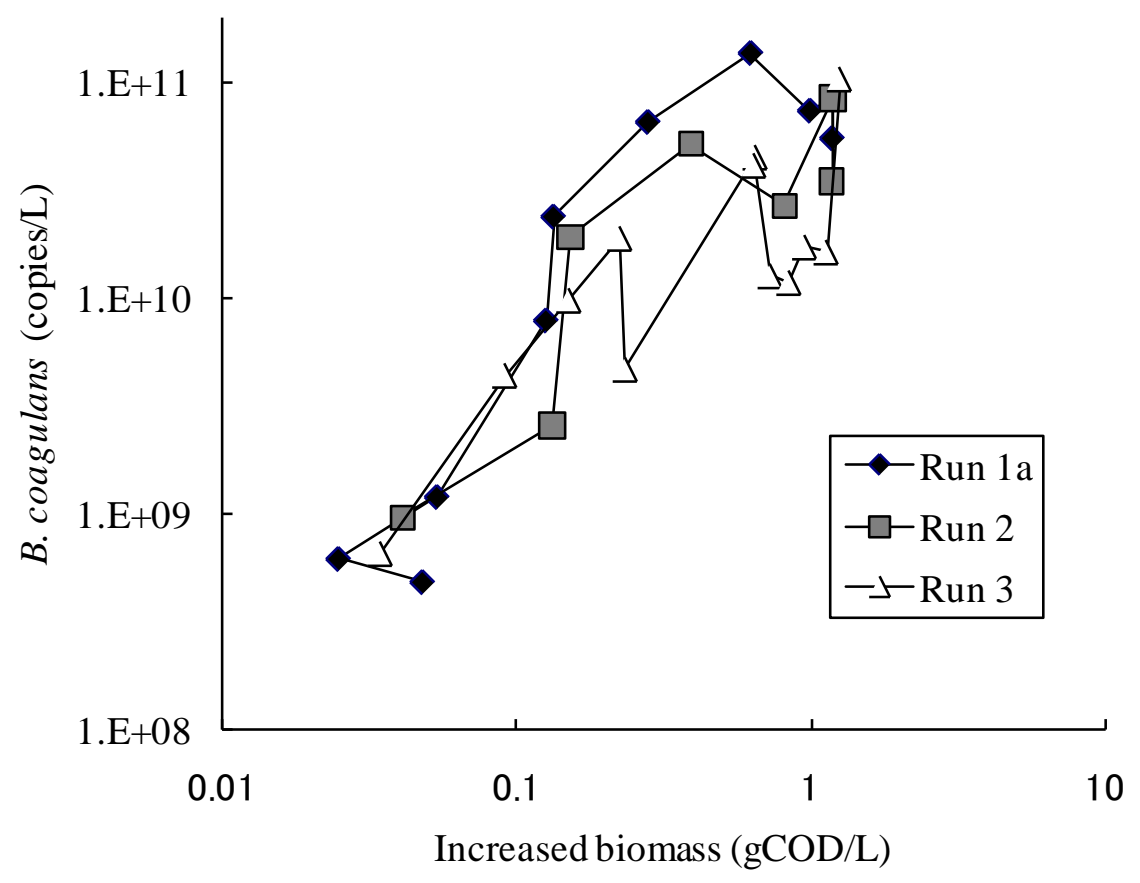

Figure 4 Relationship between increased biomass and real-time PCR analysis 

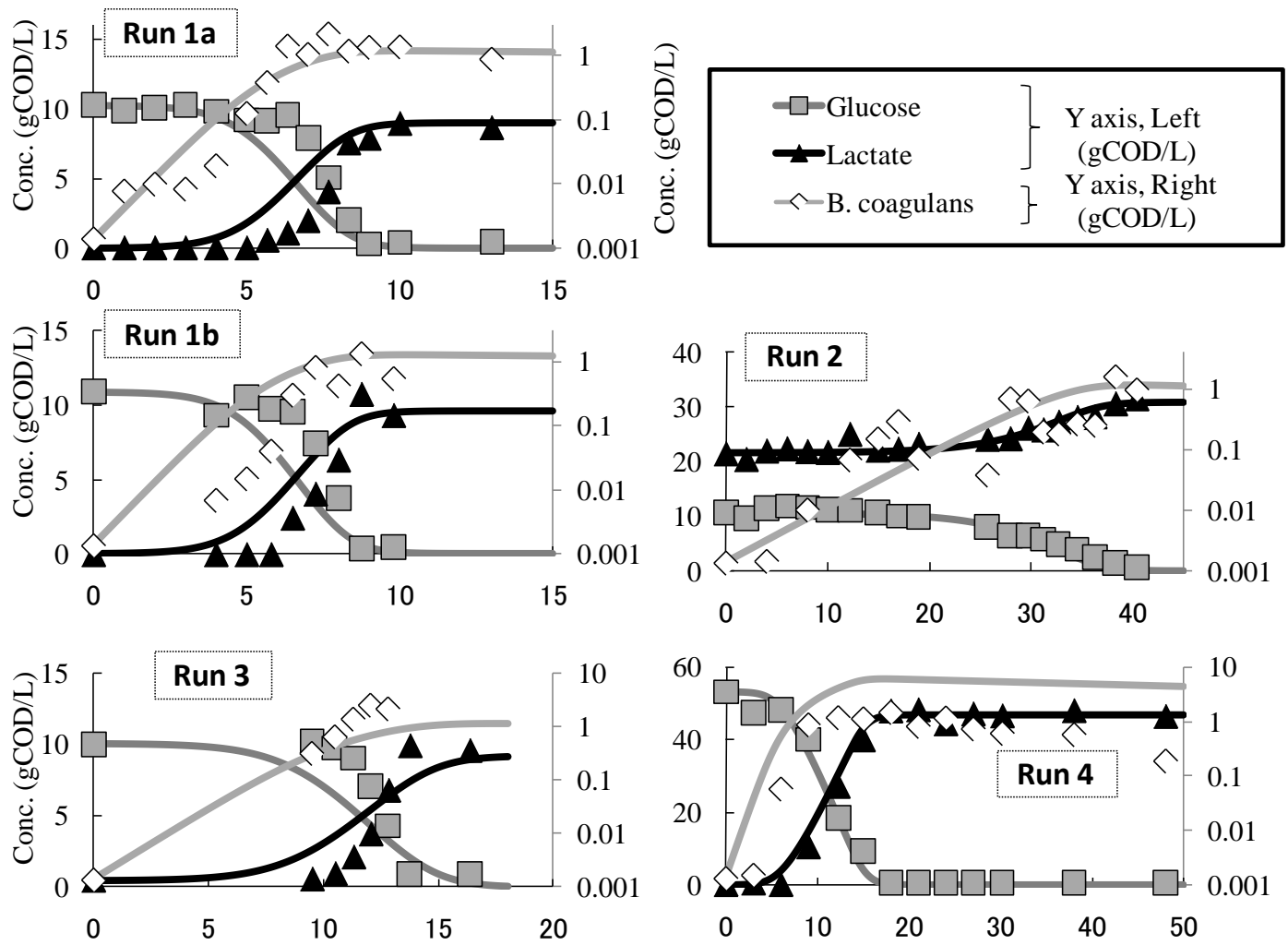

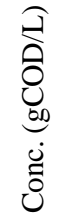
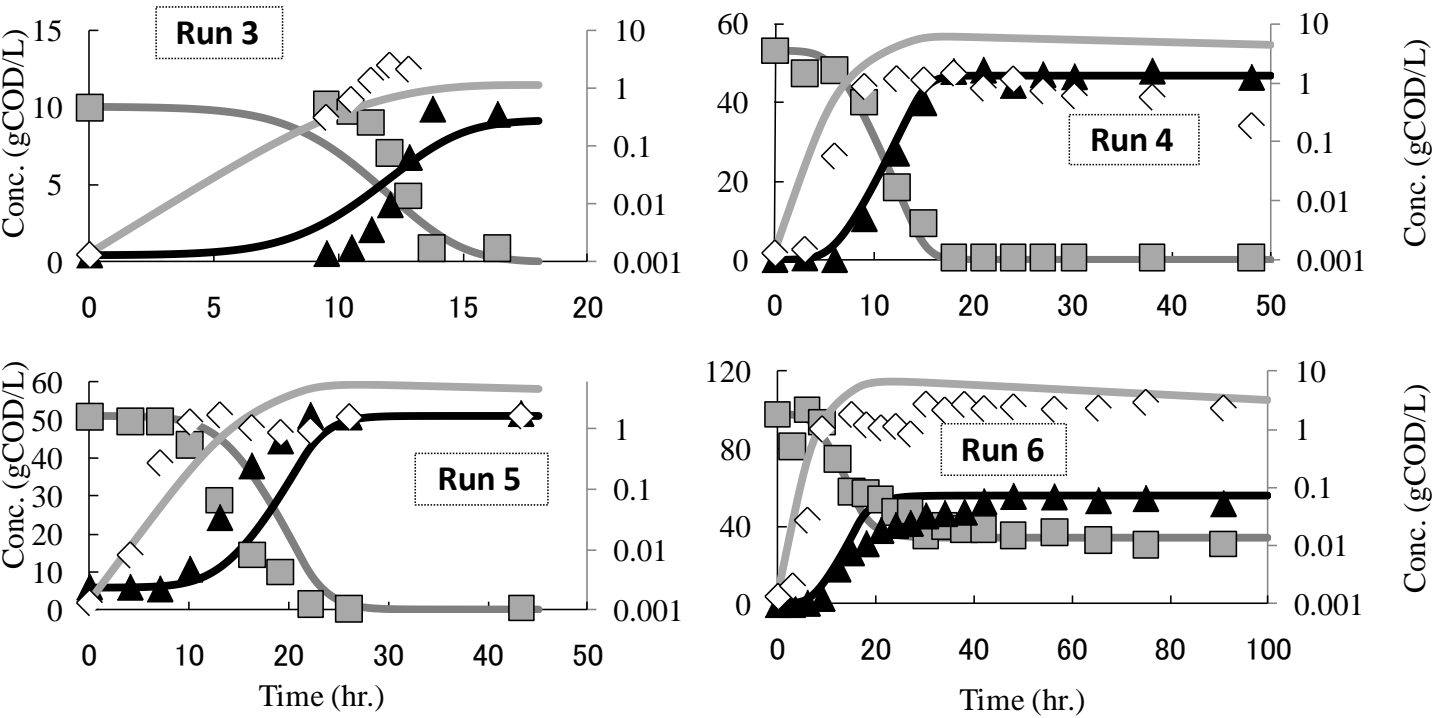

Figure 5 Time courses of the experimental and calculated results in Run 1 Run 6. Plots are experimental data and lines are simulated results. 

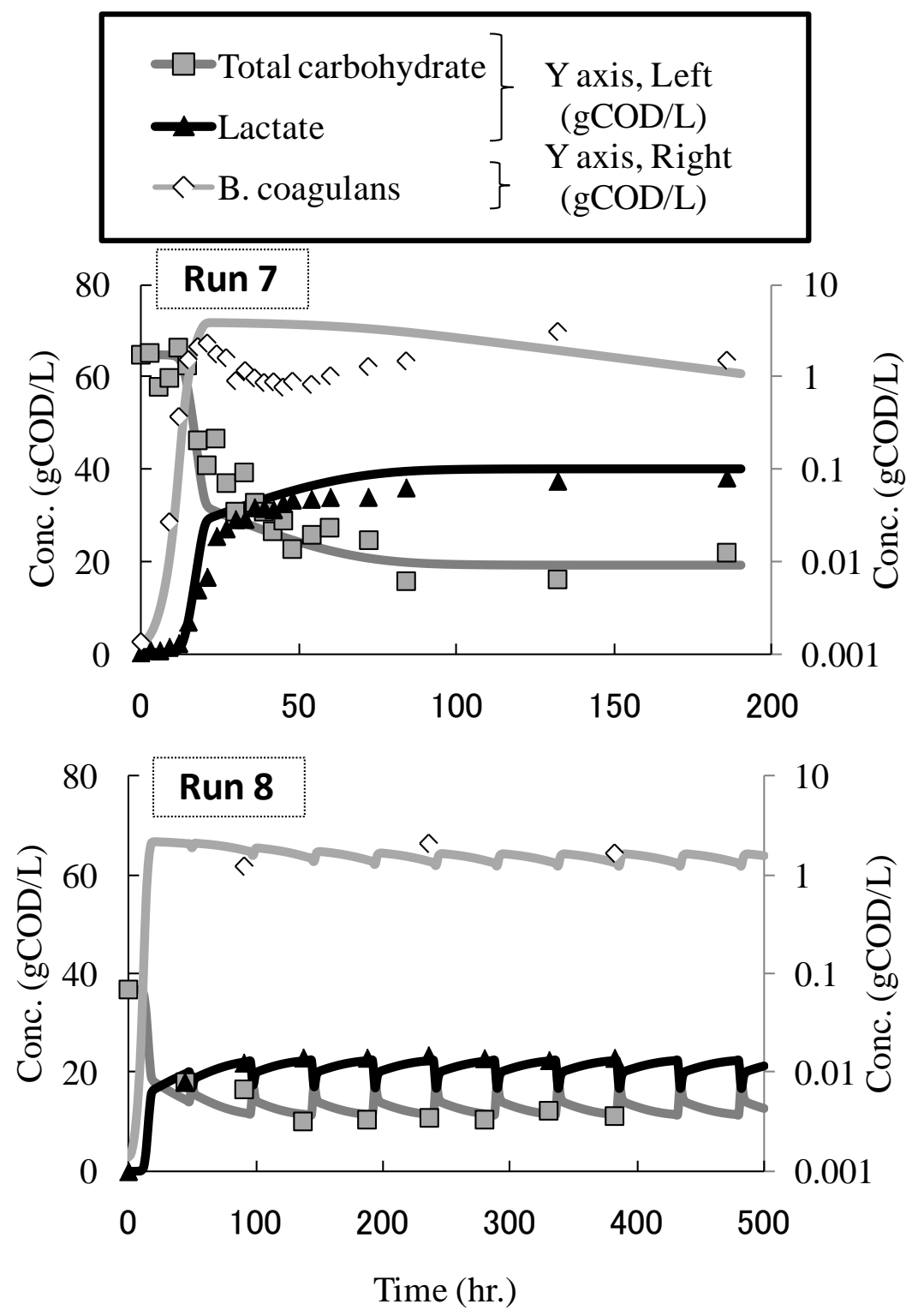

Figure 6 Time course of the experimental and calculated result in Run 7 and Run 8. Plots are experimental data and lines are simulated results. 